Horizons philosophiques

\title{
La question des valeurs dans Totalité et infini de Lévinas
}

\section{Francis Careau}

Volume 12, numéro 2, printemps 2002

Valeurs et modernité

URI : https://id.erudit.org/iderudit/801206ar

DOI : https://doi.org/10.7202/801206ar

Aller au sommaire du numéro

\section{Éditeur(s)}

Collège Édouard-Montpetit

\section{ISSN}

1181-9227 (imprimé)

1920-2954 (numérique)

Découvrir la revue

Citer cet article

Careau, F. (2002). La question des valeurs dans Totalité et infini de Lévinas. Horizons philosophiques, 12(2), 33-44. https://doi.org/10.7202/801206ar d'utilisation que vous pouvez consulter en ligne.

https://apropos.erudit.org/fr/usagers/politique-dutilisation/ 


\section{LA QUESTION DES VALEURS DANS TOTALITÉ ET INFINI DE LÉVINAS}

En ces temps de lutte au terrorisme qui prend bien souvent l'allure de chasse aux sorcières, la philosophie d'Emmanuel Lévinas sur la guerre et l'altérité dans son œuvre Totalité et Infini invite à la réflexion dans un sentiment d'urgence. Tout au long de sa vie, le penseur a continuellement dissocié la morale de la politique, jugeant cette dernière comme une mise en scène figeant l'être dans une totalité, menant parfois à la fatalité des rapports belliqueux commandés par une logique du pouvoir et une liberté inconséquente parce que sans bornes. Lévinas considère que le pouvoir, le concept spécifique du politique, est fondamentalement meurtrier de l'Autre. C'est d'ailleurs à partir d'une présentation du phénomène de la guerre que Lévinas amorce sa réflexion au sujet de la problématique des valeurs :

La guerre ne se range pas seulement - comme la plus grande - parmi les épreuves dont vit la morale. Elle la rend dérisoire. L'art de prévoir et de gagner par tous les moyens la guerre - la politique - s'impose, dès lors, comme l'exercice même de la raison. La politique s'oppose à la morale, comme la philosophie à la naïveté.1.

Cette pensée du fondement de la guerre est à ce point importante, car elle critique un même mouvement de totalisation rencontré dans la métaphysique occidentale, de Leibniz à Hegel. La thèse principale du livre de Lévinas réside dans une quête de l'altérité, c'està-dire de l'extériorité, ne se réduisant pas à une totalité ou un système clos. En effet, jamais le Même et l'Autre ne sauraient être comme deux plans ontologiques relatifs et d'un ordre similaire, s'opposant dans un système qui fait un tout. Par sa nature propre, l'altérité est dépassement, transcendance. Cette conception de l'altérité ouvre la voie à une lecture métaphysique de l'éthique.

Pour contrer une logique du pouvoir entretenue dans la sphère du politique, Lévinas va ouvrir un espace à la relation à Autrui dans l'éthique, en misant sur le concept de justice en tant qu'impossibilité

1. E. LÉVINAS, Totalité et Infini, Essai sur l'extériorité, Martinus Nijhoff, La Haye, (1961) $2^{\mathrm{e}}$ édition, 1965, p. IX. 
du meurtre. Ainsi, les philosophies du pouvoir dégénèrent souvent en philosophies de l'injustice, parce qu'elles accordent une grande valeur à la liberté ontologique plutôt qu'à la justice telle que rencontrée dans l'éthique et la métaphysique. En arrière plan de cette thèse, c'est la conception heideggerienne de la liberté qui est ici critiquée par Lévinas. Le philosophe va développer une éthique de la responsabilité de l'agent moral, divergeant sur ce point d'une morale pratique axée par exemple sur la prudence (stoïcisme) ou d'une morale à finalité esthétique. Tout en étant formulée à l'intérieur de l'expérience, cette responsabilité agit comme une rupture par rapport à la totalité en dirigeant son regard vers l'infini. L'infini de la responsabilité s'exprime dans le jugement, non pas celui de la fin de l'histoire, mais dans l'engagement personnel, dans la parole. Ce jugement se produit dans l'instant, marquant ainsi une forme de discontinuité du temps de la vie. Enfin, la responsabilité s'inscrit dans la parole :

dans le discours je m'expose à l'interrogation d'Autrui et cette urgence de la réponse - pointe aiguë du présent - m'engendre pour la responsabilité; comme responsable je me trouve ramené à ma réalité dernière ( $p .153)$.

Nous voulons refaire ce chemin du rapport métaphysique et éthique dans Totalité et Infini, en nous centrant sur la problématique des valeurs. Nous allons présenter d'abord le rapport entre langage et valeur, suivi du thème de la valeur et de l'existence, du désirable et du désiré, de la communauté, de la temporalité et de l'au-delà du visage d'autrui.

\section{Valeur et langage}

La question des valeurs est difficile à aborder à partir de Totalité et Infini, car l'ouvrage ne développe pas ici à proprement parler une éthique des valeurs. On dénombre d'ailleurs très peu d'occurrences du terme "valeur». Cela pourrait s'expliquer par le fait que, par exemple, d'emblée l'œuvre du langage apparaît comme "valeur toujours positive" (p.47). Est-ce à dire qu'une éthique de la discussion remplace une théorie des valeurs? Dit autrement, on ne s'attarderait pas à dresser une liste des valeurs, parce qu'elles se résumeraient toutes à une valeur principale : le dialogue. Dans ce contexte, le langage sert de lieu pour entrer en contact avec la nudité du visage d'autrui, c'està-dire la manifestation primordiale d'autrui. Par le concept de visage, autrui représente à la fois l'extériorité, la transcendance et 
l'infini qui se révèlent à moi. Or l'ambiguïté propre au problème des valeurs, c'est que tout en apportant certains éléments qui, nous y reviendrons, traitent du rapport entre éthique et ontologie, on s'aperçoit que l'épiphanie du visage transcende le niveau des valeurs. En effet, "la présentation de l'être dans le visage n'a pas le statut d'une valeur" (p.177), parce que cette manifestation du visage ne saurait tolérer l'ambivalence entre le vrai et le faux sousentendue par toute valeur. En d'autres mots, par son visage, autrui s'exprime dans une relation d'authenticité qui dépasse le registre consensuel et relatif des valeurs. En effet, l'altérité, c'est-à-dire l'êtrepour-autrui ne s'appuie pas, selon Lévinas, sur des valeurs préétablies, si ce n'est la bonté du moi : «Être-pour-autrui, ne doit pas suggérer une finalité quelconque et n'implique pas la position préalable ou la valorisation d'une je ne sais quelle valeur. Être pour autrui - c'est être bon» (p.239). Est-ce à dire que cette éthique nie le fondement de toute valeur? Nous ne le croyons pas. Le concept de valeur intervient à quelques endroits de Totalité et Infini et ce, en jouant un rôle parfois très positif et déterminant.

\section{Valeur et existence}

Le thème de la valeur est abordé, entre autres, dans le rapport de la vie avec la distinction entre l'essence et l'existence :

La vie est une existence qui ne précède pas son essence.

Celle-ci en fait le prix; et la valeur, ici, constitue l'être. La réalité de la vie est déjà au niveau du bonheur et, dans ce sens, au delà de l'ontologie. Le bonheur n'est pas un accident de l'être, puisque l'être se risque pour le bonheur ( $p .84)$.

Ainsi, la vie est déjà engagée dans le registre de la valeur, par les aspirations du moi et sa recherche du bonheur. II ne saurait y avoir d'exister pur, d'être pur. Cela conduirait à ce que Lévinas appelle l'ataraxie. Au contraire, la vie humaine est caractérisée par le bonheur comme accomplissement et condition de l'activité. Alors seulement, le bonheur culminera en jouissance, véritable «frisson du moi». Cette notion de jouissance désigne le plaisir que le moi peut avoir de toutes choses du domaine empirique. Ainsi, la particularité du bonheur de la jouissance façonnera la personnalité de l'être : «Jouir sans utilité, en pure perte, gratuitement, sans renvoyer à rien d'autre, en pure dépense - voilà l'humain» ( $p .107)$. On pourra dire aussi que la subjectivité réside dans les variantes personnelles apportées par la jouissance et le bonheur. Ce qui fait le moi, la personnalité, tient dans le 
caractère propre de la jouissance qui n'est ni biologique, cela serait réducteur, ni sociologique, cela conduirait à une pensée de la totalité.

Or, c'est pour éviter la totalisation que Lévinas envisage non pas l'être à partir d'une quelconque ontologie (à la manière de l'ensemble de la tradition occidentale), mais l'étant dans une théorie des valeurs, une axiologie. Ce n'est donc pas ontologiquement que la personnalité sera définie, mais axiologiquement, avec le thème du bonheur : "Le surgissement de soi à partir de la jouissance et où la substantialité du moi est aperçue non pas comme un sujet du verbe être, mais comme impliqué dans le bonheur ne relevant pas de l'ontologie, mais de l'axiologie - est l'exaltation de l'étant tout court" (p.91-92). Ainsi, ce qui forge l'identité n'est pas ontologique, mais éthique. Cette thèse est très forte et montre toute l'originalité de cette pensée. L'étant ne sera alors pas envisagé à l'intérieur d'une participation à l'être dans une antinomie entre l'être et l'étant, mais plutôt évalué par rapport au concept de bonheur. En bref, pour Lévinas, le moi ne réside ni dans l'être, ni dans la raison. Dès le départ, le moi, par le langage, attribue une valeur au monde. Cette donation, d'une portée axiologique, est déjà présente dans la vision et la jouissance. Que le fait de vivre soit perçu par certains comme heureux ou malheureux, agréable ou pénible, le moi peut se retourner contre la vie, faisant toujours de celle-ci une valeur : "Toute opposition à la vie, se réfugie dans la vie et se réfère à ses valeurs. Voilà l'amour de la vie, harmonie préétablie avec ce qui va seulement nous arriver" (p.118). Ainsi cet amour de la vie ne représente pas un souci d'être, mais le bonheur d'être.

La philosophie de Lévinas renverse le modèle classique faisant précéder l'éthique de l'ontologie : "Au dévoilement de l'être en général, comme base de la connaissance et comme sens de l'être, préexiste la relation avec l'étant qui s'exprime; au plan de l'ontologie, le plan éthique» (p.175). C'est dans le langage, c'est-à-dire dans l'expression comme don de soi, que l'être manifeste sa portée éthique, sa bonté. L'être se déploie par la parole, son verbe est nécessairement social et juste. Le langage invite au transcendant et en ce sens, il marque un bris dans la continuité de l'être. L'intériorité de l'être est, en effet, bousculée par la parole de l'autre scandant la différence. Autrui m'offre une résistance, de façon positive. Ce primat de l'axiologie sur l'ontologie dans cette définition de la personne s'explique par le recours à la notion heideggerienne de l'ustensile. En effet, Lévinas rappelle que le processus d'attribution de la valeur à l'être est actif, à la manière d'une possession, d'une prise, remplissant ainsi une 
fonction d'ustensile. Parce que la chose n'est pas originellement identique, substance, elle s'exprime dans son existence :

La fonction d'ustensile, comme la valeur que portent les choses, ne s'impose pas à la conscience spontanée comme la substance, mais comme l'un des attributs de ces êtres. L'accession aux valeurs, et l'usage et la manipulation et la manufacture reposent sur la possession, sur la main qui prend, qui acquiert, qui amène chez soi (p.136).

Or, suite à ces quelques exemples de propos sur les valeurs dans Totalité et Infini, que peut-on affirmer de la théorie lévinasienne des valeurs? Essayons d'abord d'interpréter cette philosophie à partir des questions propres à l'axiologie. Dans un premier temps, si on considère la valeur comme ce qui doit-être, c'est-à-dire l'opposé au domaine de l'être, on rencontre une telle conception chez Lévinas. Nous l'avons mentionné, l'éthique précède l'ontologie; l'étant ne se définit pas seulement par sa substance, mais aussi par sa possibilité de choisir et surtout, sa bonté. En fait, ce qui dépasse le fait d'être, c'est le caractère authentique de l'extériorité où la valeur est accordée à l'insaisissable, à l'infini, plutôt qu'à l'être. C'est seulement alors, dans cette tension vers l'infini que peut émerger le vrai : “L'aspiration à l'extériorité radicale, appelée pour cette raison métaphysique, le respect de cette extériorité métaphysique qu'il faut, avant tout, "laisser être» - constitue la vérité» ( $p$. xvii). Cette tension vers l'infini qui brise la totalité, le Même, est qualifiée de Désir métaphysique.

\section{Le désirable et le désiré}

Avec le thème du Désir, on parvient à une autre conception courante de la valeur comme propriété du désirable et non du désiré. En effet, tandis que le désiré se réfère uniquement à un sujet, à un agent moral, le désirable pousse l'audace jusqu'à tenter de déterminer des valeurs qui seraient partagées par tous. Ainsi, le désiré devrait être surpassé afin de régler le problème du solipsisme (il n'y a d'autre réalité que le je). À ce titre, Lévinas résout la question du solipsisme par sa conception du langage : "La relation du Même et de l'Autre - ou métaphysique - se joue originellement comme discours, où le Même, ramassé dans son ipséité de “je" - d'étant particulier unique et autochtone - sort du soi» (p.9). Seul l'être pensant, capable de parole, peut éprouver l'altérité. Or, suivant la 
problématique de la valeur en tant que désirable, doit-on ranger le Désir du côté du désiré ou du désirable? L'auteur distingue clairement le Désir du Désiré. En effet, il affirme que le Désiré consiste dans l'invisible, c'est-à-dire le transcendant, l'infini, l'au-delà de l'opposition entre la lumière et la noirceur. Le Désiré est le terme jamais atteint du Désir. Ce Désir, métaphysique, parce qu'il exprime la transcendance, surpasse la totalité en recherchant l'absolument autre. II ne s'agit pas d'un retour, mais plutôt d'un appel vers un "pays étranger». De plus, on insiste sur le fait que le Désir métaphysique ne tient nullement dans une sorte de besoin qui serait satisfait lorsque épanché : «Notons encore la différence entre besoin et Désir. Dans le besoin, je puis mordre sur le réel et me satisfaire d'assimiler l'autre. Dans le Désir, pas de morsure sur l'être, pas de satiété, mais avenir sans jalons devant moi" (p.89). Le Désir métaphysique n'est jamais comblé. D'ailleurs, ce caractère exprime le refus de la totalisation et son trait non égocentrique. Il est intéressant de constater que Lévinas se méfie du concept de liberté, qu'il associe à l'identification du Même, en privilégiant le Désir comme ouverture à l'extériorité :

A la théorie, comme intelligence des êtres, convient le titre général d'ontologie. L'ontologie qui ramène l'Autre au Même, promeut la liberté qui est lidentification du Même, qui ne se laisse pas aliéner par l'Autre. Ici, la théorie s'engage dans une voie qui renonce au Désir métaphysique... (p.13).

De même, la morale ne se développe pas par la liberté de l'agent, mais au contraire, elle implique une remise en question de cette liberté jugée par l'agent comme étant égoïste :

La morale commence lorsque la liberté, au lieu de se justifier par elle-même, se sent arbitraire et violente. La recherche de l'intelligible, mais aussi la manifestation de l'essence critique du savoir, la remontée d'un être en deçà de sa condition commence du même coup (p.56).

L'éthique consiste alors dans la réflexion critique du Même stimulée par la présence d'Autrui. Cette remise en question, par la raison, amène le moi à entrevoir la justice comme face-à-face avec Autrui, dans un rapport de dépendance et d'indépendance. Ce geste s'accomplit dans le langage, qui permet la relation pacifique avec Autrui où Même et Autre se rencontrent dans le Désir métaphysique. La pensée de Lévinas se situe donc en marge de tout un pan de la tradition philosophique occidentale qui entretient, dans son ontologie, l'idée d'une réduction de l'Autre au Même. Ainsi, pour contrebalancer 
le processus d'identification au Même, le philosophe suggère que la relation avec Autrui (un étant) précède l'ontologie (compréhension de l'être).

L'apparition de l'Infini comme Désirable dans le fini, c'est-à-dire le moi, a lieu dans ce que Descartes avait nommé l'idée d'Infini. Cet espace où se déploie le Désir, a un fondement résolument moral, car «posséder l'idée de l'infini, c'est déjà avoir accueilli Autrui» (p.66). De plus, le Désir de l'infini ne correspondant nullement au registre du manque, s'inscrit plutôt dans le cadre du désintéressement : «Désir parfaitement désintéressé - bonté» (p. 21). Le Désir métaphysique sera alors pleinement ouverture vers Autrui, dans un geste de générosité où on fait don à l'autre. Ce rapport éthique entre le Même et l'Autre s'élabore dans le discours. En effet, le visage d'autrui s'exprime, se manifeste, m'enseigne. Accepter le messager d'autrui, c'est posséder l'idée de l'infini. Les caractéristiques de l'idée de l'infini sont la séparation, l'intériorité, la vérité et le langage. La séparation tient dans la transcendance d'autrui. L'être est alors unique. L'intériorité tient dans la dimension personnelle accordée au temps. L'être se refuse alors à la totalisation. La vérité et le langage tiennent dans le partage et la confrontation avec Autrui de l'idée d'infini. De même, vérité et justice seront des expressions du Désir, tandis que le bonheur sera le lieu propre de la jouissance. La où le politique a comme objectif d'assurer le bonheur de tous par l'égalité, la religion encourage et alimente le Désir. Alors seulement, par la religion, la vérité pourra surgir dans le discours et dépasser ainsi la simple rhétorique par la justice vue comme reconnaissance d'autrui en tant que guide.

\section{La communauté}

Dans le langage, le rapport entre les interlocuteurs est d'emblée éthique, il trace un espace commun de signification. Avec le langage, une communauté peut s'établir, par la structure du don et de la possession en commun. C'est dans l'acte de communication que la vérité pourra advenir et non pas dans un quelconque élan mystique, solitaire. Le vrai se recherche donc en société. On peut approcher du vrai par l'éducation où autrui fait figure de maître. Ainsi, ma liberté se borne à l'autorité et au savoir du maître, qui lui-même est dépassé par l'infini. II est alors aisé de constater à quel point Autrui me transcende, comment il est métaphysique. En bref, le concept de Désir domine toute la réflexion lévinasienne de l'extériorité, car il rend possible la conscience morale. 
Le pluralisme de la communauté ne se réduit nullement à une multiplicité numérique. Ce pluralisme est éthique, car il impose une attitude réflexive à l'égard d'Autrui. Cette attitude est "déjà qualifiée», c'est-à-dire qu'elle n'est jamais neutre. Dès l'origine on peut éprouver, par exemple, de l'amour ou de la haine pour quelqu'un. Ainsi, considérer Autrui comme un autre, de façon qualifiée, nous ramène possiblement à l'ordre de la valeur. L'une des manifestations les plus évidentes de l'extériorité serait, selon Lévinas, rencontrée dans le désir sexuel. Or, le désir sexuel n'est ici qu'un aspect de la mission de la communication permettant que la communauté puisse s'instaurer. Alors, la société se fonde sur le langage, qui est une mise en commun d'un monde. Ce monde avait été préalablement possédé par le moi, dans un élan identitaire et égoïste. Par un événement ontologique, le moi s'est d'abord déterminé comme être fini et séparé. En habitant le monde, en établissant une demeure, le moi a illustré sa dimension «économique».

La manifestation d'autrui se produit fondamentalement dans son visage. Cela invite à une relation, à un discours d'origine. Le visage sensible d'autrui est le premier mode d'apparaître de l'autre, bien qu'il ne soit pas le seul. Par son visage, l'autre présente la nudité de son regard, sa fragilité, la pitié qu'il inspire. Dans sa misère, l'autre m'invite à me servir positivement de ma liberté dans une attitude de bonté. L'épiphanie du visage ne tient pas dans une sorte de dévoilement de l'intériorité. Au contraire, ce geste est un appel à l'espace public, à la société, parce qu'il s'accomplit dans le langage et que la parole annonce toujours la présence d'un tiers. On est ici aux antipodes de la conception infernale de l'altérité telle que développée par Sartre dans Huis clos. En effet, de façon pessimiste Sartre entrevoyait que l'intervention d'un tiers amenait nécessairement un conflit de volontés, c'est-à-dire des disputes de pouvoir. Tandis que chez Lévinas, le visage d'autrui expose toute la magnificence de l'humanité, c'est-àdire la fraternité. La valeur du visage d'autrui réside dans le fait qu'il offre la possibilité d'entrevoir la transcendance, l'ouverture vers l'infini. Cet espace propre à la transcendance est caractérisé par la paix. Autrui m'incite à la non-violence, parce que nulle lutte de pouvoir n'est possible, Autrui ne fait que demander au moi de justifier sa liberté par la responsabilité. Autrui est, à ce titre, le premier enseignement de la personne. Alors, se responsabiliser veut dire tourner la volonté du moi vers la raison, dans le langage, qui est, par essence, éthique. La volonté concerne le processus identitaire du moi. 


\section{Conscience de la mort et temporalité}

Le terme de la volonté figure dans la mort. La conscience du dernier moment instaure une temporalité dans la vie du moi. Le temps va alors apparaître comme un ajournement, un délai. La réflexion sur la mort annonce et oblige à s'attarder à sa propre mort. On pourrait alors reformuler le cogito cartésien : je pense donc je sais que je vais mourir. D'ailleurs, combien de philosophes n'ont-ils pas affirmé que philosopher consistait à apprendre à mourir? La peur de sa propre mort doit se transmuer en un événement très riche de sens, le signe d'une transcendance. La mort comme fin de la volonté renvoie vers ce qui me dépasse, à l'altérité. Pour retrouver un sens derrière l'inéluctable de notre destin, Lévinas suggère l'existence pour Autrui, le renforcement du Désir, l'exercice altruiste de la bonté. Vivre dans une dimension temporelle instaurée par la perspective de la mort devrait amorcer une attitude de patience, d'espoir, détroussant la volonté du moi de son caractère égoïste, par le Désir métaphysique d'autrui.

\section{L'au-delà du visage d'autrui}

Enfin, la notion de visage d'autrui annonce son propre dépassement par la thématique de l'au-delà du visage. Avec la présentation de cette section (chapitre IV) de Totalité et Infini, nous pourrons aller au cœur de ce que nous croyons être les différentes valeurs de Lévinas. En effet, si l'on considère les valeurs comme ce qui réalise la nature propre de l'agent moral, un second niveau semble compléter conclure la réflexion lévinasienne sur l'extériorité. Le premier niveau, étudié précédemment, concernait le moment originel de la rencontre avec Autrui. On a vu que, s'établissant dans le discours, cette rencontre se révélait nécessairement éthique, qu'elle invitait à la justice et à la responsabilité. Ce moment, que l'on pourrait qualifier de formation de la conscience, accompagne le processus identitaire du moi. Or, autrui, par l'expression de son visage, engage le moi à l'altérité. II s'agit, selon nous, de la conception primordiale de l'extériorité selon Lévinas, telle que nous l'avons présentée jusqu'à maintenant, que l'on peut aisément considérer comme une valeur. II nous semble, toutefois, qu'un second moment complète cette réflexion sur l'origine de l'altérité. Cet au-delà du visage d'autrui exprime une nouvelle facette du Désir qui tente de rétablir une certaine forme de continuité. En effet, nous avons vu que la conscience de la mort instituait de façon dramatique, une temporalité dans le moi. Ainsi, afin de redonner du 
sens à cette vie qui n'est qu'achèvement et sursis, le moi va orienter son regard vers le visage d'autrui. C'est précisément lors de cette orientation du moi vers le visage d'autrui, qu'un événement sans aucune commune mesure va se produire. Va apparaître, par-delà cette épiphanie, le signe d'un espoir, d'un avenir, d'une transcendance. De nouvelles valeurs, l'amour, la fécondité et la fraternité, devraient alors parfaire et accomplir la nature de l'agent moral.

L'éthique culminerait ainsi dans l'amour conjugal. Cet amour revêt un caractère ambigu, car on ne peut ni l'associer à l'érotisme vu comme besoin, ni au Désir métaphysique. On constate donc que l'amour conjugal, l'Éros, recherche Autrui dans sa faiblesse, veut lui porter secours. La transcendance du visage tient dans la quête de l'Aimée, dans ses traits féminins mystérieux et cachés : «le mouvement de l'amant devant cette faiblesse de la féminité, ni compassion pure, ni impassibilité, se complaît dans la compassion, s'absorbe dans la complaisance de la caresse" (p. 235). II y a donc une épiphanie secrète du visage qui consiste dans la fragilité et la grâce de la féminité appelant à la douceur. C'est probablement ici, avec les propos sur le féminin, que surgit le plus clairement la perspective esthétique de cette philosophie. Or, l'Éros est attiré par le caché vu comme à-venir. Éros transcende le visage parce qu'il est tourné vers le futur. L'Éros s'extériorise dans la volupté du rapport asocial des amants qui ne tolèrent pas la présence de tiers. Autrement dit, le langage de l'amour conjugal est ici exclusif, c'est-à-dire non communautaire. On pourrait alors envisager que l'amour révélerait un égoïsme partagé. Par contre, il n'est pas sourd à l'appel d'une nouvelle expression de l'extériorité : la volonté de se réaliser dans l'enfant. La fécondité agit ici comme valeur. L'enfant assure une sorte de survie au moi, tout en faisant de ce nouveau moi un autre. Ainsi dirigée vers le futur, la fécondité ouvre un rapport au temps infini. Le don de la vie est une nouvelle occasion d'agir sous le critère de la bonté. Par la reproduction, le Désir métaphysique effectue sa tâche la plus noble, soit d'engendrer le Désir. Ainsi, Autrui n'est pas le terme ultime du Désir; celui-ci s'accomplit dans un mouvement se surpassant, la «bonté de la bonté», c'est-à-dire la fécondité.

Le thème de la fécondité suggère à la fois une visée égoïste dans l'espoir de la survie du moi et à la fois le lieu de l'intersubjectivité, de la communauté. À l'origine, l'amour était vu comme asocial, car il excluait le tiers. Pour sa part, le rapport entre le père et le fils débouche sur un renouveau de la société. Dans une première étape, 
l'enfant va s'identifier comme Même, en retrouvant son trait d'unicité dans le fait qu'il a été "élu», c'est-à-dire choisi par son père. Le rapport filial sous-entend à la fois une continuité et une rupture. Dans une deuxième étape, l'enfant va se tourner vers Autrui, son frère. Par l'aspect égalitaire qu'elle favorise, la fraternité inaugure le rapport social. De fait, lorsque le visage d'autrui m'apparaît comme celui d'un frère, le lien social peut s'établir. La fraternité est ainsi une valeur exceptionnelle, parce que constitutive d'un lien social; elle permet l'égalité, la justice, la responsabilité et la générosité.

\section{En guise de conclusion}

Lévinas use de prudence à employer le concept de valeur dans Totalité et Infini. Nous croyons que cette attitude, plutôt timorée, témoigne peut-être d'une crainte d'un relativisme de nature subjectiviste. En effet, l'auteur pourrait laisser entendre que chaque valeur est propre à l'agent moral et ainsi on perdrait tout critère de vérité. Mais nous avons vu à quel point la vérité pouvait jaillir du discours. Non seulement la parole est ici une valeur, il est évident que ce programme encourage plusieurs autres valeurs. On pense, entre autres, à l'idée de justice, de responsabilité, d'amour ou de fécondité. Nous avons montré qu'en considérant deux moments propres au statut de l'extériorité, le concept de visage d'autrui et le dépassement du visage, le philosophe exploite clairement différentes valeurs. Les valeurs les plus fortes annoncées sont celles de l'amour conjugal, de la paternité, de la filiation et de la fraternité. Cette dernière valeur, l'amour entre frères, figure à la base de l'établissement de la société et rend possible l'avènement de la paix. La guerre doit alors être vue comme le fruit des hommes aliénés.

\section{Francis Careau \\ Université du Québec à Montréal}




\section{Bibliographie sélective sur l'éthique de Lévinas}

LÉVINAS, Emmanuel, Totalité et infini : essai sur l'extériorité, La Haye, Martinus Nijhof, (1961) deuxième édition 1965, $284 \mathrm{p}$.

LÉVINAS, Emmanuel, Le temps et l'autre, Paris, Presses Universitaires de France, (1979) septième édition 1998, 91 p.

LÉVINAS, Emmanuel, Altérité et transcendance, France, Fata Morgana, 1995, $182 \mathrm{p}$.

LÉVINAS, Emmanuel, Entre nous : essais sur le penser-à-l'autre, Paris, Librairie générale française, (1991) 1993, $252 \mathrm{p}$.

LÉVINAS, Emmanuel, Autrement qu'être : ou Au-delà de l'essence, Paris, Librairie générale française, 1990, $286 \mathrm{p}$. 